\title{
Attentional cartography: mapping the distribution of attention across time and space
}

\author{
J. Eric T. Taylor ${ }^{1} \cdot$ David Chan ${ }^{1}$. \\ Patrick J. Bennett ${ }^{2}$. Jay Pratt ${ }^{1}$
}

Published online: 12 June 2015

(C) The Psychonomic Society, Inc. 2015

\begin{abstract}
Decades of research have shown that the orienting of attention follows a reliable pattern of facilitation and then inhibition following a peripheral cue. However, the literature lacks a high-resolution spatiotemporal map of this pattern. Moreover, the use of visual placeholders to highlight potential stimulus locations is inconsistent. This is puzzling, given attention's well-known predilection for objects. In this article, we remedy these outstanding issues with a large-scale investigation charting the spatiotemporal distribution of attention. Participants detected targets presented at 121 possible locations $100,200,400$, or $800 \mathrm{~ms}$ following an uninformative peripheral cue. The cued locations were presented with or without placeholders. With placeholders, the classic pattern of early facilitation and late inhibition was observed for targets appearing within the placeholders, and the spread of inhibition was severely limited to within the placeholders. Without placeholders, we observed inhibition shortly after cue presentation, upsetting the famously reliable effect of facilitation following a cue. Moreover, inhibition spread from the cued location, unlike when placeholders were present. This investigation has produced an eminently detailed spatiotemporal map of attentional orienting and illustrated the consequences of placeholder stimuli, with surprising results.
\end{abstract}

Keywords Attentional capture - Space-based attention . Object-based attention

J. Eric T. Taylor j.eric.t.taylor@gmail.com

1 University of Toronto, 100 St. George St., Toronto, Ontario M5S 3G3, Canada

2 McMaster University, Hamilton, Ontario, Canada
For 35 years, researchers have fruitfully employed variations of the spatial cueing paradigm to learn how visual attention moves. This paradigm, designed and popularized by Posner (1980; Posner \& Cohen, 1984), uses abrupt peripheral onsets (cues) to attract attention. Shortly thereafter, a target is presented at the same location as the cue or at a different location; the difference in the reaction times (RTs) to detect targets at cued and uncued locations indexes the orienting of attention. These types of cues produce two effects: a brief period of facilitation, in which targets are detected faster at the cued than at uncued locations shortly after the cue, followed by a longer period of inhibition, during which targets are detected slower at cued than at uncued locations (Klein, 2000; Posner \& Cohen, 1984). This later inhibition is called inhibition of return (IOR; Posner, Rafal, Choate, \& Vaughan, 1985).

Measuring the effect of cues by comparing the RTs from only cued and uncued locations may not fully describe the distribution of attention, since there have been compelling demonstrations of the spatially continuous nature of facilitation and inhibition. For example, IOR spreads to locations neighboring the cue and attenuates with distance, suggesting a graded inhibition centered on the cued location (Maylor \& Hockey, 1985). In a similar study, in which stimuli were displayed on the perimeter of a circle, IOR appeared to be strongest at the location opposite the cue, and diminished around the perimeter (Pratt, Spalek, \& Bradshaw, 1999). The most detailed spatial study of IOR to date used four cue locations and 441 potential target locations (Bennett \& Pratt, 2001), producing a high-resolution map of the inhibitory aftereffect following peripheral cues. Inhibition was pronounced in the cued quadrant and spread in a Gaussian pattern, becoming facilitation in the opposite quadrant. Although the spatial resolution of this map is extreme, the researchers used only one cue-target onset asynchrony (CTOA; $800 \mathrm{~ms}$ ). Thus, we possess only a snapshot of attention's spatiotemporal 
distribution. In this article, we aimed to expand on this attentional cartography by generating a similarly high spatial resolution map of attention over multiple CTOAs.

Following the design of Posner and Cohen (1984), researchers employing variations of the spatial-cueing paradigm have typically used visual placeholders to indicate potential cue and/or target locations. The use of placeholders is so ubiquitous in studies examining the orienting of attention (cf. Wright \& Ward, 2008) that their presence is taken for granted. This is odd, for two reasons. First, consider the widely known effect that objects limit the spread of attention. For example, cueing one end of a rectangle speeds target detection at the other end, as compared to an equidistant location in a separate object (Egly, Driver, \& Rafal, 1994). Object-based effects are not limited to facilitation: Target detection on a moving object results in IOR, as though the inhibited region tracked the object through space (Tipper, Weaver, Jerreat, \& Burak, 1994). Simply put, objects alter the spatial distribution of attention. Second, some of Posner's early contemporaries employed similar cue-target designs without placeholder boxes - with contrasting results. When the cues and targets were represented by LEDs affixed to an arc perimeter, such that the cue and target locations were not confined within the contours of a box stimulus, the reliable facilitative cueing effect reversed; at CTOAs as short as $100 \mathrm{~ms}$, targets were detected more slowly at the cued locations (Berlucchi, Tassinari, Marzi, \& Di Stefano, 1989; Tassinari, Aglioti, Chelazzi, Marzi, \& Berlucchi, 1987). Given that attention operates qualitatively differently when objects are involved, it is important to examine how the presence of placeholders affects the spatiotemporal distribution of attention. Note that some examinations of the distribution of IOR have used placeholders (e.g., Maylor \& Hockey, 1985; Pratt et al., 1999), whereas others have not (Bennett \& Pratt, 2001); we aimed to disambiguate this inconsistency by manipulating the presence of placeholders in our display. Still other research has looked at IOR with and without placeholders (e.g., Birmingham \& Pratt, 2005; Klein \& McCormick, 1989), but not with short and long CTOAs, and never with the spatial resolution provided by our design (after Bennett \& Pratt, 2001). Perhaps the most interesting product of this method is that we will be able to see the distribution of attention not just at the placeholder locations, as in existing studies, but also at the regions surrounding them.

The present research is intended to provide an empirical demonstration of attentional orienting at high spatiotemporal resolution: attentional cartography. This expands on existing high-resolution spatial maps (e.g., Bennett \& Pratt, 2001) by examining the way that maps change over time. These maps have additional methodological impact as demonstrations of what happens when we do or do not use placeholder stimuli in these types of displays. To produce these maps, we conducted a large-scale experiment in the spirit of Posner's spatial-cueing paradigm, with four cue locations and 121 target locations at four CTOAs. Moreover, we manipulated the presence of placeholders at the cued locations. In doing so, we have obtained an eminently detailed map of the spatiotemporal distribution of attention.

\section{Method}

\section{Participants}

Eighty students from an introductory psychology class at the University of Toronto participated for course credit.

\section{Apparatus}

All stimuli were white on a black background. The display was divided into an invisible, square $11 \times 11$ grid, with $2^{\circ}$ of visual angle separating each intersection (see Fig. 1a). The 121 intersections of this grid were possible target locations. A central fixation square (subtending $0.75^{\circ}$ ) was surrounded by four equally spaced cue locations that were located at the corners of an invisible $12^{\circ} \times 12^{\circ}$ square. On some trials, square outline placeholder stimuli subtending $0.75^{\circ}$ were displayed at the cue locations. The target was a white dot subtending $0.5^{\circ}$, and the cue was a square outline subtending $0.75^{\circ}$, with an outline twice as thick as the placeholder stimuli (so that in the placeholder condition, one of the four placeholder stimuli appeared to thicken). The viewing distance was fixed at $44 \mathrm{~cm}$ using a chinrest. Participants made their responses on a keyboard placed directly in front of them.

\section{Procedure and design}

On every trial, the fixation appeared at the center location for $1,000 \mathrm{~ms}$. Next, a cue appeared randomly at one of the four locations for $100 \mathrm{~ms}$, whereupon a target would appear at one of the 121 possible locations $100,200,400$, or $800 \mathrm{~ms}$ after cue onset. The participants' task was to detect the target as quickly as possible by pressing the space bar. On some trials, no target would appear. On these catch trials, no response was required; they were used to discourage participants from making anticipatory responses. A response made during a catch trial was considered an error and caused a feedback tone to be presented. Participants were told to respond as rapidly as possible while minimizing errors. The intertrial interval was 1 , $000 \mathrm{~ms}$, and breaks were given periodically. Participants were told to fixate centrally throughout the experiment, and that the cue was completely uninformative. Data were collected in a single 2-h session. A closed-circuit camera was used to monitor eye movements and to ensure that participants remained fixated centrally.

Target Location was a within-subjects factor, and CTOA and Placeholder Presence were between-subjects factors, 


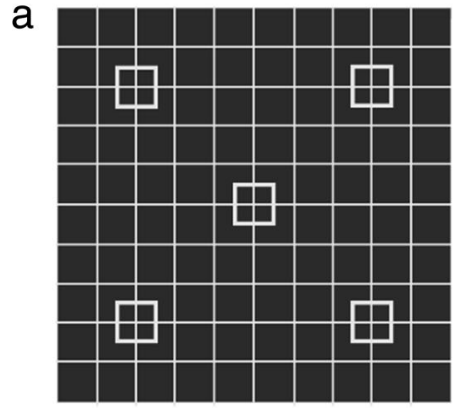

Fig. 1 a Display used in the experiment. The placeholders were present for half of the participants and were invisible for the other half, although the cues always appeared at these locations. Targets could appear at any of the 121 intersections of this invisible grid. The central box was always
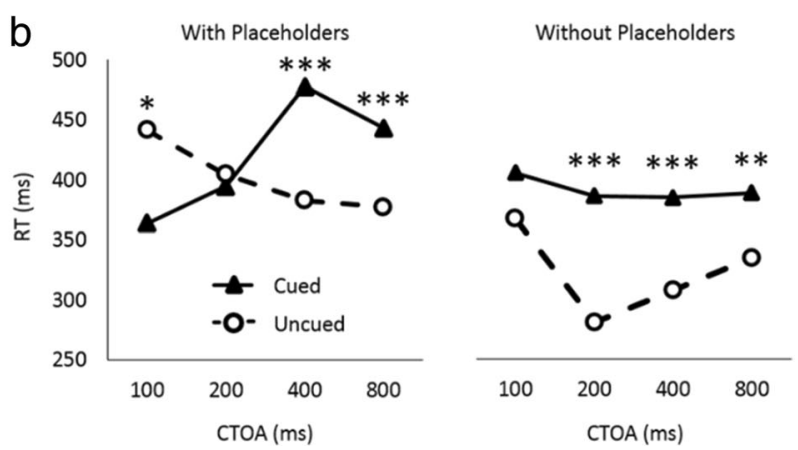

present. Placeholders are not drawn to scale. b Mean reaction times (RTs) for cued and uncued locations at different cue-target onset asynchronies (CTOAs), with and without placeholders. ${ }^{*} p \leq .05 .{ }^{* *} p \leq .01{ }^{* * *} p \leq .001$

further illustrate the effect of placeholders on the typical crossover pattern, we compared the mean cueing effects $\left(\mathrm{RT}_{\text {Uncued }}\right.$ $\left.-\mathrm{RT}_{\text {Cued }}\right)$ with and without placeholders at each CTOA with independent-samples $t$ tests. The placeholders influenced the cueing effects at 100 and $200 \mathrm{~ms}$ after the cue $(t \mathrm{~s}>2.67, p \mathrm{~s}<$ .016 ; all other $t \mathrm{~s}<0.64$, n.s.), indicating that placeholders alter the time course of attention shortly after cue presentation.

To illustrate the spread of attention over space and time, we also created scatterplots and heat maps of the data, depicted in Fig. 2. On the heat maps, the fastest of responses are represented by white squares, the slowest are represented by black squares, and the intermediate RTs are represented by shades of gray. On the scatterplots, the cued locations are highlighted by red circles, the opposite uncued locations by blue triangles, and the mean of the other uncued locations by green diamonds.

When placeholders are present, there is a steady, positive relationship between RT and distance from the cued location at the shortest CTOA. This relationship disappears at $200 \mathrm{~ms}$ and reverses at 400 and $800 \mathrm{~ms}$. Note, however, that IOR (inhibition at 400 and $800 \mathrm{~ms}$ ) is sharply confined to the cued placeholder, and to a lesser extent the other uncued placeholders: RTs to detect targets within the placeholders are dramatically lengthened relative to all surrounding locations. For a visual representation, examine the heights of the red, blue, and green points on the scatterplots in Fig. 2. They appear as outliers; RTs to targets within placeholders are highly exaggerated, indicating that they limit the spread of IOR.

When placeholders are absent, there is a negative, exponentially decreasing relationship between RTs and distance at every CTOA. These curves indicate that inhibition is strongest at the cued location and diminishes rapidly with distance. This relationship is strongest 400 and $800 \mathrm{~ms}$ after the cue. Importantly, RTs at the four potential cue locations are not unlike those at their neighbors when no placeholders are present. On the heat maps, this effect is visible in that the cued location is not confined. Rather, inhibition spreads without constraint. On 


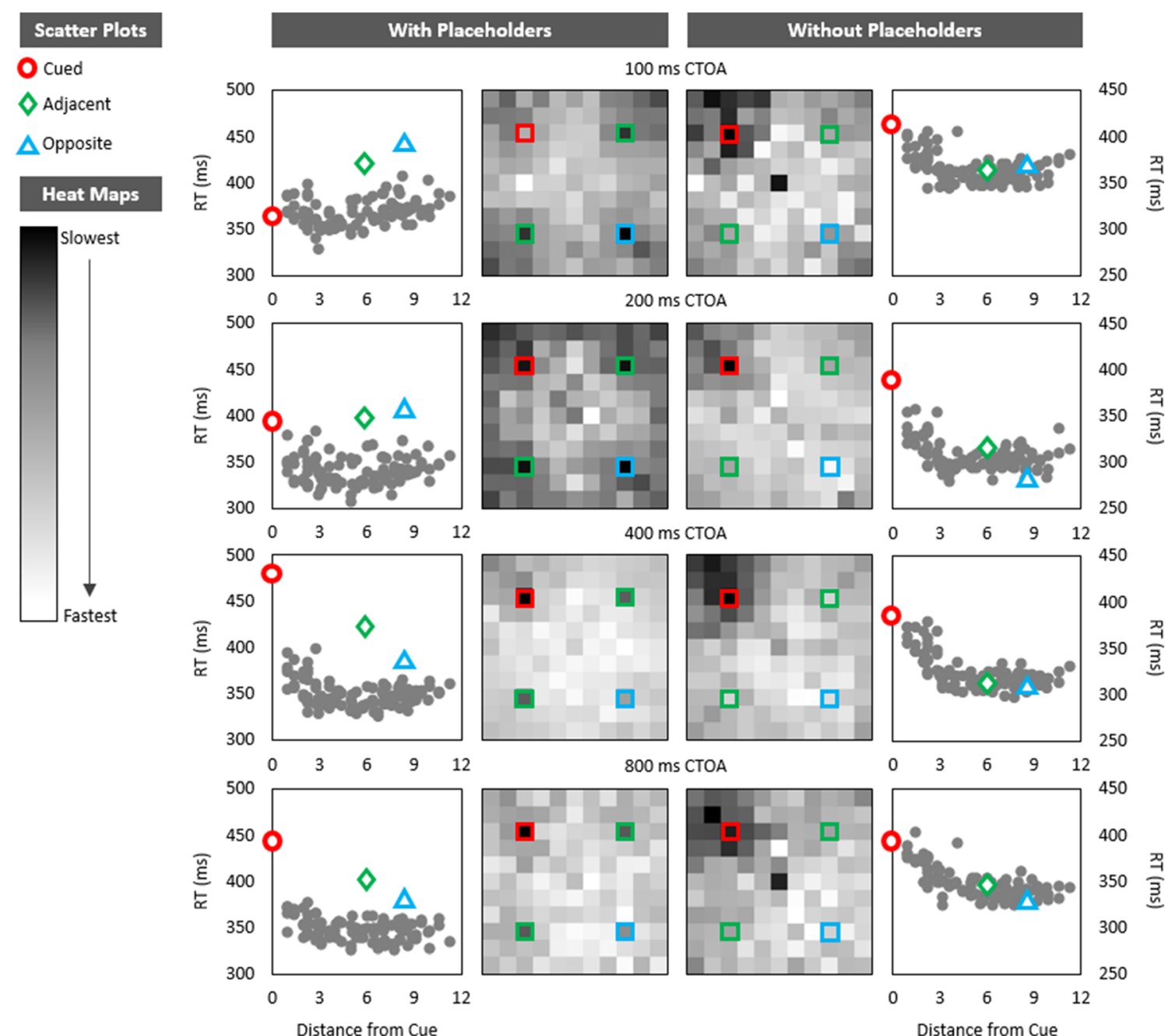

Fig. 2 Scatterplots and heat maps displaying the spatial distribution of attention at different CTOAs, with and without placeholders for cued locations. The scatterplots display the mean reaction times (RTs) at a given location as a function of how far that location was from the cue (distance is measured in grid units; each target location was $2^{\circ}$ from its neighbor). RTs for the cued location and the opposite, uncued location are shown as red circles and blue triangles, respectively; the green diamonds represent the mean RTs for the other two uncued locations. Note that the axes represent the same intervals, but different values, for placeholders

present versus absent, because RTs were slower overall when placeholders were present. For each heat map, the fastest value is white (RGB: 255, 255, 255), the slowest value is black (RGB: 0, 0, 0), and the mean RT is in mid-gray (RBG: 128, 128, 128). All other values are in intermediate shades of grays. Although the cue location was random, these heat maps have been normalized such that the cued location is always at the top left. The boxes represent the possible cue locations, and their colors correspond to the highlighted points on the accompanying scatterplots

the scatterplots, this effect is visible in that the colored points appear well within the trends.

To describe the spatial distribution of attention over time, we used an exponentially decreasing function to predict the mean RTs at different locations $\left(\mathrm{RT}=a e^{-b(\text { Distance })}+c\right)$. We conducted this regression with and without placeholders for every CTOA. The best-fit regression parameters for every condition are described in Table 1. Smaller $b$ values indicate a broad distribution of attention (a shallower bend to the curve), whereas larger $b$ values indicate a tighter distribution surrounding the cue (a steeper bend). The parameters were bound at zero, and this function cannot be increasing, which explains why the one plot without a negative relationship (placeholders present, $100 \mathrm{~ms}$ CTOA) outputs parameters describing a flat line.

\section{Discussion}

These maps illustrate the spatiotemporal distribution of covert attention elicited by peripheral cues with unsurpassed detail. Furthermore, they highlight the dramatic impact that placeholder stimuli have on this distribution. Consider the data from the cued locations. When placeholders were present, we replicated the well-known pattern of early facilitation and late inhibition. In contrast, when placeholders were absent, we 
Table 1 Regression parameters for the distribution of attention

\begin{tabular}{lllll}
\hline Cue-Target Onset Asynchrony (ms) & $a$ & $b$ & $c$ & $R^{2}$ \\
\hline With Placeholders & & & & \\
$\quad 100$ & $<0.01$ & 1.50 & 369.28 & .09 \\
200 & 53.83 & 1.72 & 341.05 & .10 \\
400 & 127.25 & 1.34 & 348.20 & .49 \\
800 & 95.27 & 1.37 & 345.61 & .44 \\
Without Placeholders & & & & \\
100 & 56.48 & 0.66 & 360.04 & .35 \\
200 & 90.39 & 0.86 & 301.25 & .52 \\
400 & 88.69 & 0.55 & 313.60 & .65 \\
800 & 67.77 & 0.46 & 337.09 & .60 \\
\hline
\end{tabular}

The parameters describe an exponentially decreasing function: $\mathrm{RT}=a e^{-}$ $b$ (Distance) $+c$. These parameters were discovered with an iterative model and were bounded as greater than or equal to zero

observed early and late inhibition at the cued location, inconsistent with the typically espoused time course of covert orienting (e.g., Posner \& Cohen, 1984). By comparing the sizes of the cueing effects at each CTOA, we found that covert orienting was dramatically altered in the presence or absence of placeholder stimuli at short (100 and $200 \mathrm{~ms}$ ) CTOAs.

Turning next to the spatial distribution of the cueing effects, our results demonstrate that the placeholders in our study acted as visual anchors that confined the spread of inhibition. This is evident on the scatterplots and heat maps, which clearly demarcate the placeholder locations as outliers relative to their surroundings. This observation is further supported by the regression analyses: The high $b$ values and low $R^{2}$ s relative to the no-placeholder condition indicate that the curve quite literally bent over backward accounting for the cued location, and that the cued locations were more prominent outliers. In contrast, when placeholders are absent, inhibition spreads over much of the visual field (replicating the findings of Bennett $\&$ Pratt, 2001, at the longest CTOA). The spread of inhibition is curvilinear, rapidly decreasing at short distances and smoothing out after about three grid units $\left(\sim 6^{\circ}\right)$. In addition to replicating existing data from Bennett and Pratt at the longest CTOA $(800 \mathrm{~ms})$, this investigation adds maps for three other CTOAs, bringing this high-resolution cartography into the temporal dimension. The results also complement similar high-resolution spatiotemporal maps of attention derived using change detection procedures, which have also revealed an opposite-hemifield boost (although those authors did not implicate IOR; Tse, Sheinberg, \& Logothetis, 2003). Furthermore, they complement new research demonstrating that structured arrays of placeholder stimuli can alter the shape of the distribution of attention (e.g., Jefferies \& Di Lollo, 2015, who showed that attention can be deployed in an annulus when the display is populated with placeholders in that shape).
The early and sustained inhibition reported in our noplaceholder condition is surprising, because it seems to contradict an established literature. However, we endorse an explanation that is consistent with the earliest descriptions of the biphasic, facilitation-then-inhibition pattern (Klein, 2004). In the seminal study of the components of visual orienting, Posner and Cohen (1984) explained the biphasic pattern of attentional orienting by positing an interaction between separate, but simultaneous, facilitative and inhibitory processes. The typical spatial-cueing task invokes an early and sustained inhibition along with an early and ephemeral facilitation that is stronger than the inhibition. Cueing effects are described by the summation of these processes, such that early CTOAs yield facilitation (on account of this being stronger than the inhibition), but later CTOAs yield inhibition, because that process outlasts the facilitation. In accordance with this model, early inhibitory cueing effects should be observed if the facilitative component can be erased or quickly terminated. For example, when observers are given a strong incentive to disengage from a peripheral cue, inhibitory cueing effects are observed at CTOAs as short as $50 \mathrm{~ms}$ (Danziger \& Kingstone, 1999). Similarly, Collie and colleagues observed facilitation by peripheral cues only when the cue presentation overlapped with the target; when the cue was flashed briefly and offset, they observed no facilitation, even at short $(<200$-ms) CTOAs (Collie, Maruff, Yucel, Danckert, \& Currie, 2000). Although Collie et al. did not comment on this finding, we propose that it can be considered as a manifestation of the aforementioned rapid disengagement: Perhaps the transient nature of those cues speeded disengagement, relative to the longer-duration cues that did elicit facilitation. Similarly, we propose that the absence of placeholders in our display made it easier or more likely for observers to disengage attention from the cued locations, expediting decay of the facilitative component. ${ }^{1}$ The corollary to this explanation is that when placeholders are present, observers default to a mode in which they anchor attention to those high-contrast contours. This seems likely, given that observers automatically allocate attention within the contours of objects' edges in well-known demonstrations of object-based attention (Egly et al., 1994). It is not clear why exactly we did not observe facilitation without placeholders, but the aforementioned studies (Collie et al., 2000; Danziger \& Kingstone, 1999), and others not showing early facilitation with placeholder stimuli (e.g., Samuel \& Kat, 2003; Samuel \& Weiner, 2001), illustrate that this violation of the typical crossover pattern cannot be the only factor in our finding.

Why, then, did placeholder boxes in our study attract such attentional priority? Perhaps because they were the only objects (besides the fixation box) in the display, observers defaulted attention to them. Another possibility is that attention has a predilection for edges, which is consistent with the

\footnotetext{
${ }^{1}$ We thank an anonymous reader for proposing this idea.
} 
object-based literature (Scholl, 2001). Placeholder stimuli also bring a lot of perceptual baggage when they are inserted into an otherwise blank display, and this complicates interpretations of the effect of such placeholders. Consider that the relative change in luminance in the display caused by the appearance of a cue or target is greater in the placeholder condition than in the no-placeholder condition. ${ }^{2}$ Thus, the observed differences between the placeholder and noplaceholder conditions could be caused by perceptual factors independent of attention (e.g., salience, sensory adaptation, etc.). This idea is also consistent with early reports of inhibitory cueing effects at short CTOAs $(200 \mathrm{~ms})$ in displays without placeholder boxes (e.g., Berlucchi et al., 1989; Tassinari et al., 1987). These researchers used LEDs as their stimuli instead of placeholder boxes. Like our no-placeholder condition, the sudden illumination of an LED would produce a much larger relative change in luminance than Posner and Cohen's (1984) brightening boxes. In any event, the present results offer a clear demonstration of the consequences of a paradigm-typical placeholder display on the covert orienting response.

Another theoretical complication produced by elements of our display is the potential confound of target eccentricity with the results. Because our goal required the examination of many target locations over a wide area, we were unable to control for target eccentricity when producing our map. We are not too concerned, though, because Bennett and Pratt (2001), after whom our display was designed, conducted a small study to examine the effect of eccentricity and found no effect with their display. They used a similar display, without cues, and targets appeared at 40 different locations along the four diagonals extending from fixation (ten locations on each diagonal). Because they found no effect of eccentricity on simple target detection with their display, and because our display (i.e., very bright stimuli on a black background) and procedure were practically identical, we are confident that there was no influence of eccentricity on our data.

By dramatically increasing the number of target locations, we produced an eminently detailed map of the orienting response. But this also had the effect of reducing the reliability of the cue stimulus. Whereas most studies use a handful of cue and target locations, making even uninformative cues somewhat reliable, the likelihood of the target appearing at the cued location in our study was low: $1 / 121$, to be exact (or a 25/121 likelihood of appearing in the cued quadrant). It is reasonable to suppose that the unreliability of the cue affected the distribution of attention (e.g., Druker \& Anderson, 2010). However, even though the likelihood of the target appearing at the cued location was very low (1/121), we still replicated the typical facilitation-then-inhibition crossover pattern at the cued versus the opposite location (see Fig. 1b) in the

${ }^{2}$ We thank an anonymous reader for proposing this idea. placeholder conditions, so it seems unlikely that the cue unreliability upset typical orienting behavior.

Because the number of trials required for sufficient power at 121 target locations was so high (>1,000 trials/participant), CTOA and placeholder presence were manipulated between subjects. Since all participants had experience with only one CTOA, the repeated temporal context might have influenced the surprising results observed in the no-placeholder condition. As we noted above, we would expect any effect of temporal context to be equipotent across placeholder conditions; given that we observed a highly paradigm-typical pattern of results in the placeholder condition, we do not expect that temporal context caused the surprising results in the noplaceholder condition. With respect to placeholder presence as a between-subjects manipulation, we wanted to ensure that participants in the no-placeholder condition never had exposure to the objects, since uncertainty in the trial-to-trial object structure can increase object-based selection (e.g., Shomstein, 2012), which may influence the orienting response.

This investigation was not the first on covert orienting to systematically manipulate the presence of placeholder stimuli. Other researchers have examined the effect that placeholder stimuli have on IOR (e.g., Birmingham \& Pratt, 2005; Jordan \& Tipper, 1998; Klein \& McCormick, 1989; McAuliffe, Chasteen, \& Pratt, 2006; McAuliffe, Pratt, \& O'Donnell, 2001; Paul \& Tipper, 2003; Pratt \& Chasteen, 2007), but only at longer $(>500-\mathrm{ms})$ CTOAs and with fewer stimulus locations (e.g., 2-8), often using multiple sequential-cueing paradigms, and never investigating cases in which a cue occurs at a placeholder location and the target does not (preventing finegrained analyses of spatial distribution). Notwithstanding, they served as a useful reference for the effect of placeholder stimuli on spatial orienting. A common thread throughout these studies is that IOR is weakened without as compared to with placeholders (Jordan \& Tipper, 1998; McAuliffe et al., 2006, with young adults; McAuliffe et al., 2001; Pratt \& Chasteen, 2007; although some have shown no differencee.g., Klein \& McCormick, 1989; McAuliffe et al., 2006, with old adults), which seems at odds with our finding of significant IOR at $200 \mathrm{~ms}$ without placeholders, versus no IOR with placeholders. We do not contend that IOR was greater without placeholders, but rather that the time course of facilitation and inhibition was altered by the placeholders' presence. Had previous investigations examined the effect of placeholders on peripheral cueing at earlier CTOAs, they might have found similar results, and if we had extended our investigation to CTOAs greater than $1,000 \mathrm{~ms}$, we might have observed greater IOR with placeholders as time progressed, as in these earlier studies. Interestingly, Birmingham and Pratt (2005) found no or minimal IOR at all without placeholders in a sequential-cueing paradigm with CTOAs between 1,000 and 3,000 ms. Given the accelerated pattern of inhibition observed in our no-placeholder condition, it may be possible that 
the IOR that we observed was short-lived and did not extend past the 800 -ms CTOA (although further investigation is warranted to resolve these differences).

The results from this large-scale, high-resolution study of the effects of peripheral cues on attention have shown the enormous effect that placeholders can have on both the time course and spatial distribution of attention. In other words, the spatiotemporal dynamics of attention are heavily influenced by something that is usually regarded as a feature of experimental design rather than an independent variable. By looking at more locations, across a larger range of time, than ever before, the present study not only highlights the critical role of placeholders, but also reconciles a longstanding discrepancy in the cueing literature and provides detailed information about how peripheral cues affect behavior over time and space. In light of the present findings, one wonders what would now be considered the "classic" pattern of cueing effects, had the early studies (Maylor \& Hockey, 1985; Posner $\&$ Cohen, 1984) not used placeholders.

Author note This research was supported by a grant from the Natural Sciences and Engineering Research Council of Canada to J.P. We also thank Brian Scholl, Matt Hilchey, James Danckert, Michael Dodd, and an anonymous reader for suggestions that improved the manuscript.

\section{References}

Bennett, P. J., \& Pratt, J. (2001). The spatial distribution of inhibition of return. Psychological Science, 12, 76-80. doi:10.1111/1467-9280.00313

Berlucchi, G., Tassinari, G., Marzi, C. A., \& Di Stefano, M. (1989). Spatial distribution of the inhibitory effect of peripheral non-informative cues on simple reaction time to non-fixated visual targets. Neuropsychologia, 27, 201-221. doi:10.1016/0028-3932(89)90172-3

Birmingham, E., \& Pratt, J. (2005). Examining inhibition of return with onset and offset cues in the multiple-cuing paradigm. Acta Psychologica, 118, 101-121. doi:10.1016/j.actpsy.2004.10.005

Collie, A., Maruff, P., Yucel, M., Danckert, J., \& Currie, J. (2000). Spatiotemporal distribution of facilitation and inhibition of return arising from the reflexive orienting of covert attention. Journal of Experimental Psychology: Human Perception and Performance, 26, 1733-1745. doi:10.1037/0096-1523.26.6.1733

Danziger, S., \& Kingstone, A. (1999). Unmasking the inhibition of return phenomenon. Perception \& Psychophysics, 61, 1024-1037. doi:10. 3758/BF03207610

Druker, M., \& Anderson, B. (2010). Spatial probability aids visual stimulus discrimination. Frontiers in Human Neuroscience, 4, 63. doi: 10.3389/fnhum. 2010.00063

Egly, R., Driver, J., \& Rafal, R. D. (1994). Shifting visual attention between objects and locations: Evidence from normal and parietal lesion subjects. Journal of Experimental Psychology: General, 123, 161-177. doi:10.1037/0096-3445.123.2.161

Jefferies, L. N., \& Di Lollo, V. (2015). When can spatial attention be deployed in the form of an annulus? Attention, Perception, \& Psychophysics, 77, 413-422. doi:10.3758/s13414-014-0790-4

Jordan, H., \& Tipper, S. P. (1998). Object-based inhibition of return in static displays. Psychonomic Bulletin \& Review, 5, 504-509. doi:10. 3758/BF03208829
Klein, R. M. (2000). Inhibition of return. Trends in Cognitive Sciences, 4, 138-147. doi:10.1016/S1364-6613(00)01452-2

Klein, R. M. (2004). Orienting and inhibition of return. In M. S. Gazzaniga (Ed.), The cognitive neurosciences (3rd ed., pp. 545559). Cambridge: MIT Press.

Klein, R., \& McCormick, P. (1989). Covert visual orienting: Hemifield activation can be mimicked by zoom lens and midlocation placement strategies. Acta Psychologica, 70, 235-250.

Maylor, E. A., \& Hockey, R. (1985). Inhibitory component of externally controlled covert orienting in visual space. Journal of Experimental Psychology: Human Perception and Performance, 11, 777-787. doi:10.1037/0096-1523.11.6.777

McAuliffe, J., Chasteen, A. L., \& Pratt, J. (2006). Object- and locationbased inhibition of return in younger and older adults. Psychology and Aging, 21, 406-410. doi:10.1037/0882-7974.21.2.406

McAuliffe, J., Pratt, J., \& O’Donnell, C. (2001). Examining locationbased and object-based components of inhibition of return in static displays. Perception \& Psychophysics, 63, 1072-1082. doi:10. 3758/BF03194525

Paul, M. A., \& Tipper, S. P. (2003). Object-based representations facilitate memory for inhibitory processes. Experimental Brain Research, $148,283-289$.

Posner, M. I. (1980). Orienting of attention. Quarterly Journal of Experimental Psychology, 32, 3-25. doi:10.1080/ 00335558008248231

Posner, M. I., \& Cohen, Y. (1984). Components of visual orienting. In H. Bouma \& D. G. Bouwhuis (Eds.), Attention and performance X: Control of language processes (pp. 531-556). Hillsdale: Erlbaum.

Posner, M. I., Rafal, R. D., Choate, L. S., \& Vaughan, J. (1985). Inhibition of return: Neural basis and function. Cognitive Neuropsychology, 2, 211-228. doi:10.1080/02643298508252866

Pratt, J., \& Chasteen, A. L. (2007). Examining inhibition of return with multiple sequential cues in younger and older adults. Psychology and Aging, 22, 404-409. doi:10.1037/0882-7974.22.2.404

Pratt, J., Spalek, T. M., \& Bradshaw, F. (1999). The time to detect targets at inhibited and noninhibited locations: Preliminary evidence for attentional momentum. Journal of Experimental Psychology: Human Perception and Performance, 25, 730-746. doi:10.1037/ 0096-1523.25.3.730

Samuel, A. G., \& Kat, D. (2003). Inhibition of return: A graphical metaanalysis of its time course and an empirical test of its temporal and spatial properties. Psychonomic Bulletin \& Review, 10, 897-906. doi:10.3758/BF03196550

Samuel, A. G., \& Weiner, S. (2001). Attentional consequences of object appearance and disappearance. Journal of Experimental Psychology: Human Perception and Performance, 27, 1433-1451. doi:10.1037/0096-1523.27.6.1433

Scholl, B. J. (2001). Objects and attention: The state of the art. Cognition, 80, 1-46. doi:10.1016/S0010-0277(00)00152-9

Shomstein, S. (2012). Object-based attention: Strategy versus automaticity. Wiley Interdisciplinary Reviews: Cognitive Science, 3, 163-169.

Tassinari, G., Aglioti, S., Chelazzi, L., Marzi, C. A., \& Berlucchi, G. (1987). Distribution in the visual field of the costs of voluntarily allocated attention and of the inhibitory after-effects of covert orienting. Neuropsychologia, 25, 55-71. doi:10.1016/0028-3932(87)90043-1

Tipper, S. P., Weaver, B., Jerreat, L. M., \& Burak, A. L. (1994). Objectbased and environment-based inhibition of return of visual attention. Journal of Experimental Psychology: Human Perception and Performance, 20, 478-499. doi:10.1037/0096-1523.20.3.478

Tse, P. U., Sheinberg, D. L., \& Logothetis, N. K. (2003). Attentional enhancement opposite a peripheral flash revealed using change blindness. Psychological Science, 14, 91-99.

Wright, R. D., \& Ward, L. M. (2008). Orienting of attention. Oxford: Oxford University Press. 